\title{
The ontogeny of P-glycoprotein in the developing human blood-brain barrier: implication for opioid toxicity in neonates
}

\author{
Jessica Lam, Stephanie Baello, Majid Iqbal, Lauren E. Kelly, \\ Patrick T. Shannon, David Chitayat, Stephen G. Matthews, and \\ Gideon Koren
}

Version Publisher's PDF

Citation Lam, Jessica, et al. "The ontogeny of P-glycoprotein in the developing (published version) human blood-brain barrier: implication for opioid toxicity in neonates." Pediatric research 78.4 (2015): 417. doi: 10.1038/pr.2015.119

Copyright/License

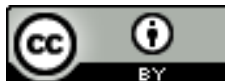
Attribution 4.0 International License. To view a copy of this license, visit https://creativecommons.org/licenses/by/4.0/.

How to cite TSpace items

Always cite the published version, so the author(s) will receive recognition through services that track citation counts, e.g. Scopus. If you need to cite the page number of the author manuscript from TSpace because you cannot access the published version, then cite the TSpace version in addition to the published version using the permanent URI (handle) found on the record page.

This article was made openly accessible by $U$ of $T$ Faculty.

Please tell us how this access benefits you. Your story matters. 


\title{
The ontogeny of P-glycoprotein in the developing human blood-brain barrier: implication for opioid toxicity in neonates
}

\author{
Jessica Lam ${ }^{1,2}$, Stephanie Baello ${ }^{3}$, Majid lqbal ${ }^{2}$, Lauren E. Kelly ${ }^{4}$, Patrick T. Shannon ${ }^{5}$, David Chitayat ${ }^{6,7}$, Stephen G. Matthews ${ }^{3}$ \\ and Gideon Koren ${ }^{1,2,4}$
}

\begin{abstract}
BACKGROUND: Neonates have been shown to have a heightened sensitivity to the central depressive effects of opioids compared to older infants and adults. The limited development of P-glycoprotein (P-gp) may limit the ability of the neonate to efflux morphine from the brain back to the systemic circulation. The objective of the study was to determine the ontogeny of P-gp in the human brain.
\end{abstract}

METHODS: Postmortem cortex samples from gestational age (GA) 20-26 wk, GA 36-40 wk, postnatal age (PNA) 0-3 mo, PNA 3-6 mo, and adults were immunostained for P-gp.

RESULTS: The intensity of P-gp staining in adults was significantly higher compared to at GA $20-26 w k(P<0.05)$, GA $36-40$ wk $(P<0.05)$, and PNA 0-3 mo $(P<0.05)$. P-gp intensity at GA 20-26wk $(P<0.05)$, GA 36-40wk $(P<0.05)$, and PNA 0-3 mo $(P<0.05)$ was significantly lower compared to at PNA 3-6 mo.

CONCLUSION: P-gp expression in the brain is limited at birth, increases with postnatal maturation, and reaches adult levels at 3-6 mo of age. Given the immaturity of blood-brain barrier (BBB) P-gp after birth, morphine may concentrate in the brain. This provides mechanistic support to life threatening opioid toxicity seen with maternal codeine use during breastfeeding.

$\mathbf{P}$ -glycoprotein (P-gp) is a membrane ATP binding cassette transporter present in many tissues such as the kidneys, liver, intestines, testes, and brain (1). Due to its strategic location on the luminal membrane of brain endothelial cells, it has been suggested that it plays an important role in limiting the entry of drugs into the brain $(2,3)$. In brain endothelial cells, a portion of the P-gps is localized in caveolae and interacts with caveolin-1 $(4,5)$. It has been shown that when these two proteins interact, P-gp activity is inhibited (6). However the localization of P-gp in caveolae and its interaction with caveolin-1 remains controversial (7) and could depend on the specific cell line and detergent used in caveolae purification methods $(8,9)$. At present, no studies have looked at caveolin-1 expression at the developing blood-brain barrier (BBB) or how its expression may modulate P-gp function at the developing BBB.

P-gp at the BBB limits the entry of substrates found the blood from moving into the brain. These substrates have to move from blood into the brain endothelium in order to be effluxed by P-gp as the substrate-binding site is in the cytoplasmic portion of P-gp. Substrates can also originate from the brain tissue and move into the brain endothelium to be effluxed by P-gp into the blood. Morphine is commonly used for the management of mild to severe pain and different experimental approaches have demonstrated that P-gp influences the distribution of morphine across the BBB (10-13), and hence its analgesic, and adverse effects.

Neonates have been shown to have a heightened sensitivity to the central depressive effects of opioids compared to older infants and adults in both animal and human studies (14-19). Furthermore, recent studies have documented that the newborn infant is sensitive to the respiratory effects of morphine when the mother is breastfeeding while taking codeine $(20,21)$. This may be attributed to the limited expression of P-gp at the BBB during the neonatal period leading to increased morphine concentration in the brain $(14,15)$. P-gp has been detected as early as embryonic day 10.5 in the mouse brain and served as the earliest marker for endothelial cell differentiation during BBB development (22). P-gp gene expression in the mouse brain is low during late gestation and reaches adult levels by 3 wk of life (22-24).

In humans, P-gp immunoreactivity was detected as early as $8-12$ wk gestation in the fetal brain with its prevalence and intensity progressively increased with advancing age (25-27). However, even at term, P-gp expression in the fetus is low compared to adults (25). In contrast, the ontogeny of P-gp in the developing human infant has not been previously studied.

The objective of this study was to quantify the ontogeny of P-gp in endothelial cells of the developing BBB. We hypothesized that P-gp expression would be low at birth and increase dramatically in the neonatal period, explaining why young infants are sensitive to the central effects of morphine during the first months of life compared to older infants and adults.

\footnotetext{
J.L. and S.B. share first authorship.

'Department of Pharmacology and Toxicology, University of Toronto, Toronto, Ontario, Canada; ${ }^{2}$ Division of Clinical Pharmacology and Toxicology, The Hospital for Sick Children, Toronto, Ontario, Canada; ${ }^{3}$ Department of Physiology, Obstetrics and Gynaecology, University of Toronto, Toronto, Ontario, Canada; ${ }^{4}$ Department of Physiology and Pharmacology, University of Western Ontario, Toronto, Ontario, Canada; ${ }^{5}$ Department of Pathology and Laboratory Medicine, Mount Sinai Hospital, Toronto, Ontario, Canada; ${ }^{6}$ Department of Obstetrics and Gynaecology, Prenatal Diagnosis and Medical Genetics Program, Mount Sinai Hospital, Toronto, Ontario, Canada; ${ }^{7}$ Division of Clinical and Metabolic Genetics, Department of Pediatrics, The Hospital for Sick Children, Toronto, Ontario, Canada. Correspondence: Gideon Koren (gkoren@sickkids.ca) 


\section{RESULTS}

The intensity of P-gp immunoreactivity progressively increased in the brain with increasing age (Figure 1). The intensity of P-gp in adults was significantly higher compared to that at 20-26wk gestation (GA 20-26wk) $(P=0.0002)$, 36-40 wk gestation (GA 36-40wk) $(P=0.0002)$, and in infants aged 0-3 mo (PNA 0-3 mo) $(P=0.0044)$. Furthermore, there was a more pronounced decrease in P-gp intensity at GA $20-26 \mathrm{wk}$ $(P=0.0011)$, GA 36-40wk $(P=0.0013)$, and PNA $0-3$ mo $(P=0.0173)$ compared to at PNA 3-6 mo. P-gp expression reaches adults levels by $3-6$ mo of age $(P=0.74)$.

\section{DISCUSSION}

P-gp expression at the BBB plays an essential role in limiting xenobiotic exposure through active efflux from the central nervous system (CNS). This is the first study examining the ontogeny of P-gp in the human BBB in the fetal, infant, and adult period. Our results indicate that the P-gp immunostaining intensity was relatively low during the fetal and newborn period, but increases significantly with postnatal maturation. By PNA 3-6 mo of life, P-gp intensity in microvessel endothelial cells of the cortex approximated that in adults. Thus, the increased sensitivity to opioids in newborn infants compared to older infants may be attributable, at least in part, to the low expression of P-gp at birth and thereby allowing opioids to concentrate in the CNS.

P-gp immunoreactivity was detected in microvessels of the cortex as early as GA 20 wk suggesting that P-gp plays an important role in neuroprotection during fetal brain

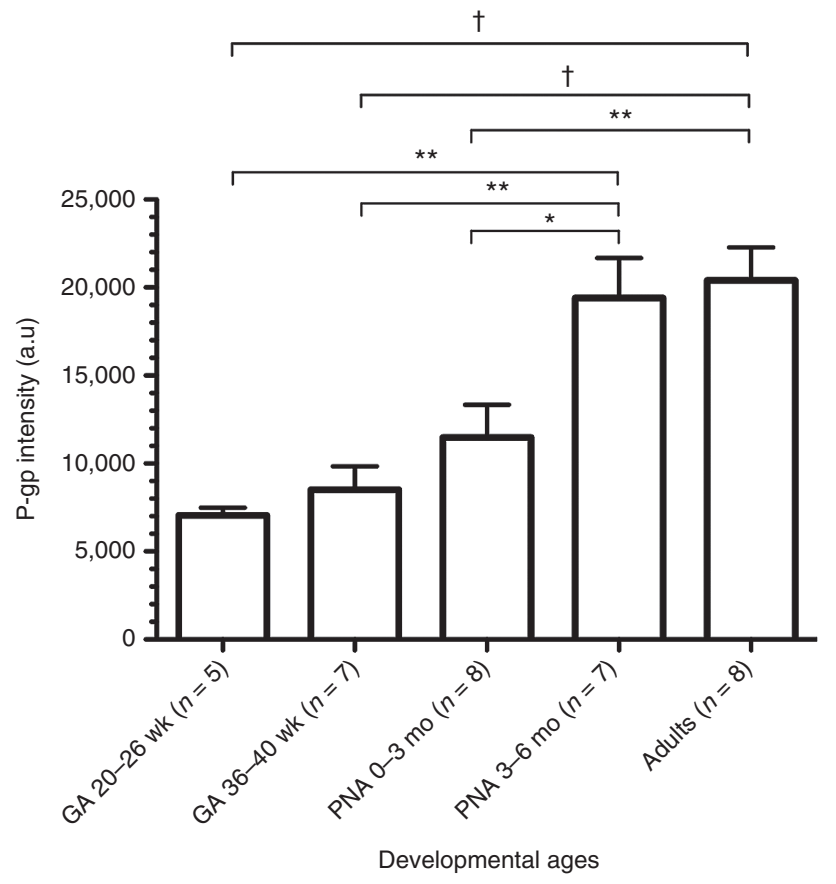

Figure 1. Intensity of P-gp at various developmental ages. Seven brain microvessels from each sample were selected and the intensity of P-gp was quantified. The intensity of P-gp progressively increased during development, reaching adult levels by 3-6 mo. Data expressed as mean \pm SEM at each developmental age. P-gp intensity was compared using an one-way ANOVA followed by the Tukey's multiple comparison test $\left({ }^{*} P<0.05,{ }^{* *} P<0.01,{ }^{\dagger} P<0.001\right)$. development, as has previously been shown in animal models (28). Previous studies in the human fetal brain detected P-gp in brain microvessels at crown-rump lengths corresponding to GA 17-24wk, serving as an early marker of BBB development $(26,27,29)$. Virgintino et al. (27) first detected P-gp as diffuse cytoplasmic labeling of the endothelial cells lining the cortical microvessels, which became linear and continuous on endothelial cell membranes at GA $22 \mathrm{wk}$. Since Virgintino et al. did not investigate the expression of P-gp in the developing human cortex at GA $20 \mathrm{wk}$, it is possible that P-gp may have already been expressed at the endothelial cells of the cortex microvessel before GA $22 \mathrm{wk}$. Similarly, the youngest human fetal brain in the study, GA $22 \mathrm{wk}$, was stained positive for P-gp (25).

P-gp immunoreactivity in human cortex was confined to brain microvessels. Levels of P-gp increase with advancing age but do not reach adult levels by term indicating further postnatal changes. By 3-6 mo of life, P-gp levels in the brain endothelial cells approximated those in the adults. This developmental pattern mimics what has been reported in the mouse brain (23). P-gp expression in the mouse brain was limited during late embryogenesis and the newborn period and increased remarkably with postnatal maturation reaching adult levels by day 21 of life (23). In humans, the intensity of P-gp immunostaining in the microvessel endothelial cell increased from GA 22 wk up to term but the P-gp intensity at term was still significantly lower when compared to adults (25).

Although the functional impact of normal developmental changes in BBB P-gp expression has not been thoroughly investigated, studies in adults have demonstrated that P-gp plays an active role in the barrier function by protecting the brain against the accumulation of toxic xenobiotics (2,30-32). Recently, it has been shown that developmental changes in $\mathrm{BBB}$ P-gp contribute to the accumulation of cyclosporine A, a P-gp substrate in the murine brain (33). Specifically, cyclosporine A accumulation within the brain was the highest in newborn mice and decreased with increasing postnatal P-gp expression (33). A study in guinea pig brain, which has a brain growth pattern that resembles human fetal development, showed that the expression of P-gp protein in the brain microvessels increases with advancing age $(28,34)$. Hence, premature and full-term neonates may be at risk for CNS drug toxicity due to the limited BBB P-gp expression in the brain microvessels. Furthermore, neonates and young infants have been shown to be more sensitive to the central depressive effects of morphine compared to older children and adults in both animal and human studies (14-19). Taken together with the findings from the present study, differences in BBB P-gp expression during brain development most likely enhance the CNS passage into the brain. As P-gp expression reaches adult levels by age 3-6 mo, older infants have the capacity to efflux morphine from the brain before it has a chance to exert its depressant effect. There are possibly other mechanisms for the increased neonatal sensitivity to opioid such as slower clearance rate of morphine and other narcotics, enhanced intestinal absorption, and higher $\mathrm{BBB}$ permeability due to other mechanisms. These mechanisms should be explored in the future to understand 
the underlying mechanisms that may contribute to drug sensitivity during the neonatal period.

There are a number of limitations to the present study that should be acknowledged. Postmortem cortex samples were used in this study. P-gp immunostaining and intensity may be modulated by the conditions that proceed at the time or after death such as hypoxia following intrauterine death, and umbilical cord or placental infarction. Intrauterine death comprises more than half of all fetal deaths (7/12). P-gp expression in murine brains was increased following hypoxia-induced treatment (35). Thus hypoxia could have artificially increased the P-gp intensity in the fetal samples. P-gp immunostaining and intensity may also be modulated by the postmortem interval. However, the postmortem interval was unknown in this study. Information regarding time from death to tissue sampling, time from sampling to tissue fixation, and method of tissue fixation were not collected. Only cortex samples were used in this study. Future studies should investigate the ontogeny of P-gp in other brain regions and astrocytes. Moreover, genetic polymorphisms, which were not assessed in this cohort, have been shown to alter P-gp expression $(36,37)$. Future studies should address the impact of genetic polymorphisms on P-gp-medicated drug transport in newborns.

A challenge with using postmortem material for immunofluorescence is tissue autofluorescence. Autofluorescence is caused by the fixation technique and the presence of fluorescent pigments in tissues. The brain has high levels of fluorescent pigment, lipofuscin. To reduce these levels, copper sulfate was applied to quench background fluorescence caused by lipofuscin (4).

Moreover, aldehyde-based fixation used to fix the brain samples forms crosslinks between proteins. This may result in inability of some protein epitopes to bind to respective antibodies. Antigen-retrieval via heated sodium citrated in conjunction with proteinase $\mathrm{K}$ digestion was used to to improve transporter epitope detection (5). This study was insufficiently powered to examine the contributions of age within the fetusinfant group, and the effects of gender differences.

In conclusion, BBB P-gp expression is incomplete in the newborn period but increases rapidly after birth and reaches adult levels by 3-6 mo. The limited BBB P-gp expression in newborns may allow drugs to concentrate in the brain, and as a consequence, lead to increased sensitivity to many drugs. Insight regarding the development of P-gp expression gained from this cohort furthers our understanding of the pharmacodynamics behind neonatal drug exposure.

\section{METHODS}

\section{Subjects and Samples}

Autopsies of adults and fetuses delivered at Mount Sinai Hospital in Toronto, Ontario, Canada were reviewed to identify cases with no history of neurological pathology, no chromosomal anomalies, normal neurological examination and a formalin fixed paraffin-embedded postmortem cortex block available for study. Fetuses of 20-26 wk gestation (GA 20-26 wk) $(n=5)$ and 36-40 wk gestation (GA 36-40 wk) $(n=7)$, representing the late second trimester and second half of the third trimester, were identified between 2010 and 2012. The fetal age was estimated on the basis of the crown-rump length and/or pregnancy records (last menstrual cycle and assessments of fetal physical maturity). In addition, adult subjects (mean age $53.6 \pm 6.5 \mathrm{y}$ old) $(n=$ 8) were identified between 2010 and 2012.
Autopsies of infants from the Department of Pathology at the London Health Science Center in London, Ontario, Canada were reviewed to identify cases with no history of neurological pathology, no chromosomal anomalies, a normal neuropathological exam and postmortem a formalin fixed paraffin-embedded postmortem cortex block. Infants aged $0-3$ mo (PNA 0-3 mo) $(n=8)$ and 3-6 mo (PNA 3-6 mo) $(n=7)$ were identified between 2009 and 2012. The infant age was estimated on the basis of the pregnancy records and neonatal physical maturity. Table 1 summarizes the characteristics and causes of death from the autopsy reports. This study was approved by the Research Ethics Board of the Mount Sinai Hospital in Toronto, Canada, University of Toronto in Toronto, Ontario, Canada, University of Western Ontario in London, Ontario, Canada and the London Health Science Center in London, Ontario, Canada. All the Research Ethics Board granted a waiver of consent for next of kin in this study based on the following two necessary conditions: (i) There is a public interest in this research while protecting the privacy of individuals and (ii) There are adequate safeguards to protect the privacy of individuals. It was also considered that given the subject group was deceased individuals, contacting the families would cause distress. The data collected in this study were coded and analyzed anonymously. No personal identifiers were collected.

\section{Immunohistochemistry}

Coronal sections of $5-\mu \mathrm{m}$ thickness were cut from the formalin fixed paraffin embedded postmortem cortex blocks and mounted on positively charged microscopic slides. The samples were deparaffinized using xylene and rehydrated through a series of graded ethanol. The sections were rinsed (three times) with phosphate-buffered saline (PBS). The sections were incubated with $10 \mathrm{nmol} / \mathrm{l}$ sodium citrate with $0.05 \%$ Tween $20\left(\mathrm{pH} 6.0\right.$ at $97^{\circ} \mathrm{C}$ ) for $30 \mathrm{~min}$. The sections were incubated at room temperature in ammonium chloride (50 nmol/l; $\mathrm{pH} 7.4)$ with PBS for $10 \mathrm{~min}$. The sections were blocked in $5 \%$ normal goat serum $(1 \mathrm{~h}$; room temperature). Subsequently, the sections were incubated with the mouse monoclonal anti-P-gp antibody D-11 (diluted 1:50; Santa Cruz, Dallas, TX) and double immunolabeled with rabbit anti-laminin antibody (diluted 1:50; Sigma-Aldrich, Oakville, Ontario, Canada) overnight at $4{ }^{\circ} \mathrm{C}$. After rinsing (five times) with PBS, the sections were incubated ( $1 \mathrm{~h}$; room temperature) with Alexa Fluor 488 goat anti-mouse (diluted 1:1,000; Life Technologies, Burlington, Ontario, Canada) and Alexa Fluor 594 chicken anti-rabbit (diluted 1:1,000; Life Technologies) for P-gp and laminin detection, respectively. The sections were rinsed and incubated with $0.5 \mu \mathrm{g} / \mathrm{ml}$ 4',6-diamidino-2-phenylindole (DAPI) (Life Technologies; $10 \mathrm{~min}$; room temperature). Sections were rinsed with PBS and distilled water before being incubated in copper sulfate (1 $\mathrm{mmol} / \mathrm{l}$; pH 5.0; $30 \mathrm{~min}$; room temperature). Sections were then rinsed with distilled water, PBS and mounted with 1,4-diazabicyclo[2.2.2] octane. A negative control section was prepared by omitting the mouse monoclonal anti-P-gp antibody. Results from the negative control confirmed the specificity of the antibody staining for P-gp.

\section{Spinning Disc Confocal Microscopy and P-gp Quantification}

Sections were viewed using the WaveFX Spinning Disc Confocal System by Quorum (Guelph, Ontario, Canada) with optimized Yokogawa CSU X1, Hamamatsu EM-CCD digital camera Image EM (C9100-13), and Leica DM16000B inverted research grade motorized microscope run by the Volocity 5.2.2 Acquisition software (Improvision, PerkinElmer, MA). On the tripled-immunolabeled section, a sequential scan procedure was applied during image acquisition of the three channels, Alexa Fluor 488 (excitation at $488 \eta \mathrm{m}$ and detection range 500-535 $\eta \mathrm{m}$; green fluorescence), Alexa Fluor 594 (excitation at $594 \eta \mathrm{m}$ and detection at $617 \eta \mathrm{m}$; red fluorescence) and DAPI (excitation at 385-400 $\eta \mathrm{m}$ and detection range $450-470$ $\eta \mathrm{m}$; blue fluorescence). All images were acquired using constant imaging parameters for each channel (green channel: exposure time$489 \mathrm{~ms}$, sensitivity- 255 , power $90 \%$; red channel: exposure time$689 \mathrm{~ms}$, sensitivity- 255 , power- $90 \%$, and blue channel: exposure time- $697 \mathrm{~ms}$, sensitivity- 255, power-90\%). Confocal images were taken at $1 \mu \mathrm{m}$ intervals through the $\mathrm{z}$-axis of the section covering in total $6 \mu \mathrm{m}$ in depth. Images of $0.5 \times 0.5 \mathrm{~mm}$ were recorded digitally and stored as TIFF files with the Volocity 5.2.2 Acquisition software (Improvision, PerkinElmer). Confocal imaging was performed 


\section{Articles | Lamet al.}

Table 1. Characteristics of fetal, infant, and adult samples

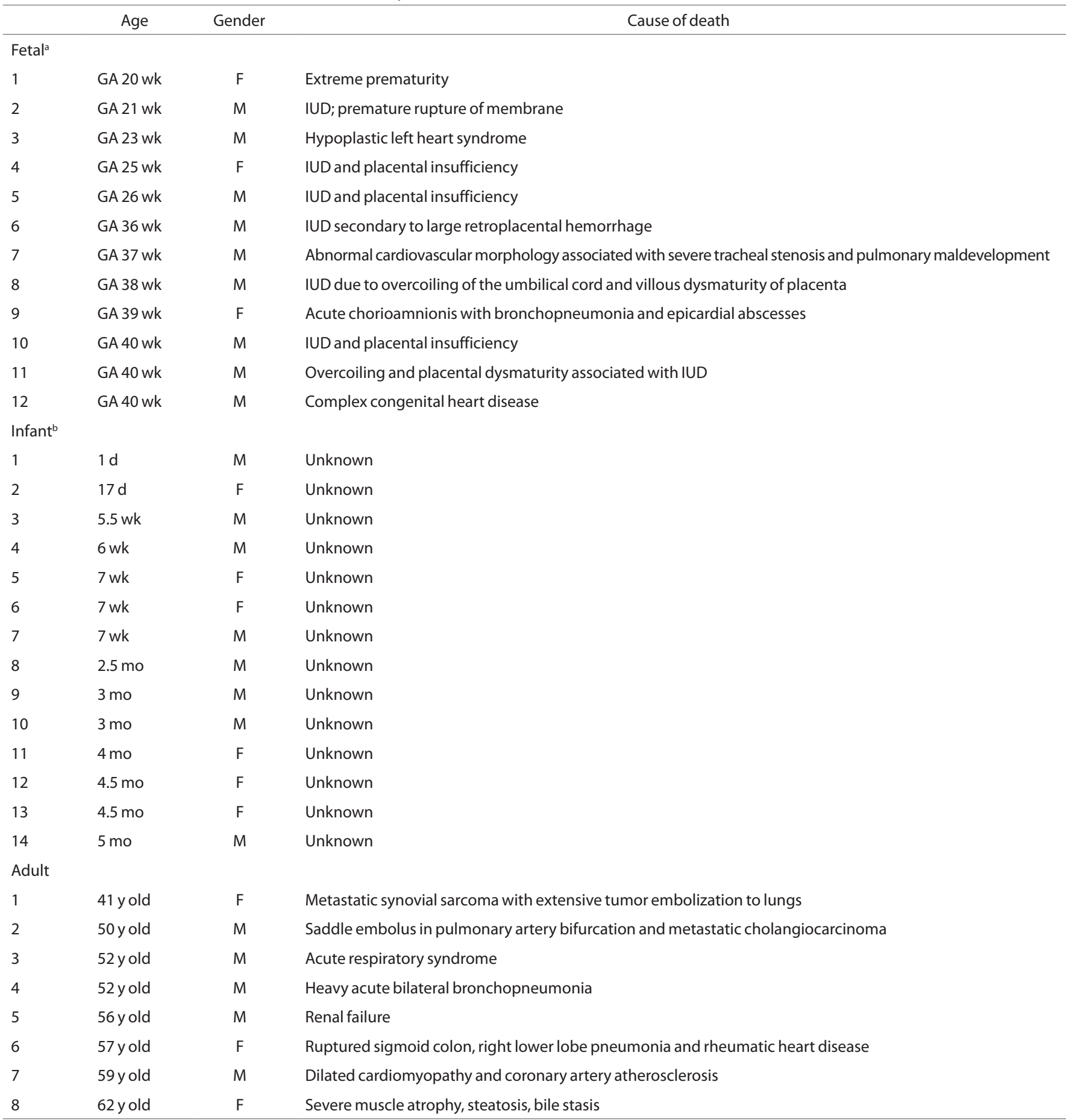

aFetal age estimated based on crown-rump length and/or pregnancy records (last menstrual cycle and assessments of fetal physical maturity. ${ }^{b}$ nfant age estimated based on pregnancy records and neonatal physical maturity. IUD, intrauterine death.

at The Lunenfeld-Tanenbaum Research Institute in Mount Sinai Hospital, Toronto, Canada.

P-gp intensity was measured in seven brain microvessels from each section using Volocity Quantification 6.3 (Improvision, PerkinElmer). Brain microvessels for measurement were selected using the following criteria: (i) contained unbranched segments of at least $50 \mu \mathrm{m}$ in length, (ii) appeared undamaged in transmitted light and fluorescence modes, and (iii) the diameter of the microvessel was between $6-10 \mu \mathrm{m}$. The selected microvessel was outlined manually using the region of interest tool to measure the average P-gp intensity of vessel.
The fluorescence intensity was corrected for background. Slides were processed by one investigator. A second investigator viewed the images to ensure that the microvessels selected met these criteria. The level of intensity for each vessel measured by Volocity Quantification 6.3 correlated with P-gp expression; as P-gp was more expressed, the intensity measured increased.

\section{Statistical Analysis}

Statistical analysis was performed using SPSS (IBM, version 20, Somers, NY). The intensity of P-gp among the developmental age 
groups was compared using a one-way ANOVA followed by a Tukey's multiple comparison test.

\section{ACKNOWLEDGMENT}

The authors would like to thank Ryszard Bielecki for his excellent technical assistance with the spinning disc confocal microscope.

\section{AUTHOR CONTRIBUTIONS}

J.L, L.E.K., and G.K. designed the study. J.L., L.E.K., P.S., and D.C. collected the samples. J.L., S.B., and M.I. collected the data. J.L., S.B., S.G.M., and G.K. analyzed the data. J.L. wrote the manuscript. All authors critically reviewed the manuscript and provided important intellectual content prior to submission.

\section{STATEMENT OF FINANCIAL SUPPORT}

This work was supported by the Canadian Institute for Health Research Grant FRN-84220 (S.G.M).

Disclosure: The authors have no conflict of interest to declare.

\section{REFERENCES}

1. Thiebaut F, Tsuruo T, Hamada H, Gottesman MM, Pastan I, Willingham MC. Cellular localization of the multidrug-resistance gene product P-glycoprotein in normal human tissues. Proc Natl Acad Sci USA 1987;84:7735-8.

2. Schinkel AH, Smit JJ, van Tellingen $\mathrm{O}$, et al. Disruption of the mouse mdr1a P-glycoprotein gene leads to a deficiency in the blood-brain barrier and to increased sensitivity to drugs. Cell 1994;77:491-502.

3. Schinkel AH. P-Glycoprotein, a gatekeeper in the blood-brain barrier. Adv Drug Deliv Rev 1999;36:179-94.

4. Schnell SA, Staines WA, Wessendorf MW. Reduction of lipofuscin-like autofluorescence in fluorescently labeled tissue. J Histochem Cytochem 1999;47:719-30.

5. Lyck L, Dalmau I, Chemnitz J, Finsen B, Schrøder HD. Immunohistochemical markers for quantitative studies of neurons and glia in human neocortex. J Histochem Cytochem 2008;56:201-21.

6. Jodoin J, Demeule M, Fenart L, et al. P-glycoprotein in blood-brain barrier endothelial cells: interaction and oligomerization with caveolins. J Neurochem 2003;87:1010-23.

7. Hinrichs JW, Klappe K, Hummel I, Kok JW. ATP-binding cassette transporters are enriched in non-caveolar detergent-insoluble glycosphingolipid-enriched membrane domains (DIGs) in human multidrug-resistant cancer cells. J Biol Chem 2004;279:5734-8.

8. Radeva G, Perabo J, Sharom FJ. P-Glycoprotein is localized in intermediate-density membrane microdomains distinct from classical lipid rafts and caveolar domains. FEBS J 2005;272:4924-37.

9. Gaus K, Rodriguez M, Ruberu KR, et al. Domain-specific lipid distribution in macrophage plasma membranes. J Lipid Res 2005;46:1526-38.

10. Dagenais C, Graff CL, Pollack GM. Variable modulation of opioid brain uptake by P-glycoprotein in mice. Biochem Pharmacol 2004;67:269-76.

11. Zong J, Pollack GM. Morphine antinociception is enhanced in mdrla gene-deficient mice. Pharm Res 2000;17:749-53.

12. Letrent SP, Pollack GM, Brouwer KR, Brouwer KL. Effects of a potent and specific P-glycoprotein inhibitor on the blood-brain barrier distribution and antinociceptive effect of morphine in the rat. Drug Metab Dispos 1999;27:827-34.

13. Thompson SJ, Koszdin K, Bernards CM. Opiate-induced analgesia is increased and prolonged in mice lacking P-glycoprotein. Anesthesiology 2000;92:1392-9.

14. Kupferberg HJ, Way EL. Pharmacologic basis for the increased sensitivity of the newborn rat to morphine. J Pharmacol Exp Ther 1963;141:105-12.

15. Bragg P, Zwass MS, Lau M, Fisher DM. Opioid pharmacodynamics in neonatal dogs: differences between morphine and fentanyl. J Appl Physiol (1985) 1995;79:1519-24.

16. Rai A, Bhalla S, Rebello SS, Kastrissios H, Gulati A. Disposition of morphine in plasma and cerebrospinal fluid varies during neonatal development in pigs. J Pharm Pharmacol 2005;57:981-6.
17. Bouwmeester NJ, Hop WC, van Dijk M, Anand KJ, van den Anker JN, Tibboel D. Postoperative pain in the neonate: age-related differences in morphine requirements and metabolism. Intensive Care Med 2003;29:2009-15.

18. Way WL, Costley EC, Leongway E. Respiratory sensitivity of the newborn infant to meperidine and morphine. Clin Pharmacol Ther 1965;6:454-61.

19. Koren G, Butt W, Chinyanga H, Soldin S, Tan YK, Pape K. Postoperative morphine infusion in newborn infants: assessment of disposition characteristics and safety. J Pediatr 1985;107:963-7.

20. Madadi P, Ross CJ, Hayden MR, et al. Pharmacogenetics of neonatal opioid toxicity following maternal use of codeine during breastfeeding: a casecontrol study. Clin Pharmacol Ther 2009;85:31-5.

21. Lam J, Kelly L, Ciszkowski C, et al. Central nervous system depression of neonates breastfed by mothers receiving oxycodone for postpartum analgesia. J Pediatr 2012;160:33-7.e2.

22. Qin Y, Sato TN. Mouse multidrug resistance $1 \mathrm{a} / 3$ gene is the earliest known endothelial cell differentiation marker during blood-brain barrier development. Dev Dyn 1995;202:172-80.

23. Tsai CE, Daood MJ, Lane RH, Hansen TW, Gruetzmacher EM, Watchko JF. $\mathrm{P}$-glycoprotein expression in mouse brain increases with maturation. Biol Neonate 2002;81:58-64

24. Ek CJ, Wong A, Liddelow SA, Johansson PA, Dziegielewska KM, Saunders NR. Efflux mechanisms at the developing brain barriers: ABCtransporters in the fetal and postnatal rat. Toxicol Lett 2010;197:51-9.

25. Daood M, Tsai C, Ahdab-Barmada M, Watchko JF. ABC transporter (P-gp/ABCB1, MRP1/ABCC1, BCRP/ABCG2) expression in the developing human CNS. Neuropediatrics 2008;39:211-8.

26. Schumacher U, Mollgård K. The multidrug-resistance P-glycoprotein (Pgp, MDR1) is an early marker of blood-brain barrier development in the microvessels of the developing human brain. Histochem Cell Biol 1997;108:179-82.

27. Virgintino D, Errede M, Girolamo F, et al. Fetal blood-brain barrier P-glycoprotein contributes to brain protection during human development. J Neuropathol Exp Neurol 2008;67:50-61.

28. Iqbal M, Gibb W, Matthews SG. Corticosteroid regulation of P-glycoprotein in the developing blood-brain barrier. Endocrinology 2011;152: 1067-79.

29. van Kalken CK, Giaccone G, van der Valk P, et al. Multidrug resistance gene (P-glycoprotein) expression in the human fetus. Am J Pathol 1992;141:1063-72.

30. Schinkel AH, Wagenaar E, van Deemter L, Mol CA, Borst P. Absence of the mdrla P-Glycoprotein in mice affects tissue distribution and pharmacokinetics of dexamethasone, digoxin, and cyclosporin A. J Clin Invest 1995;96:1698-705.

31. Schinkel AH, Wagenaar E, Mol CA, van Deemter L. P-glycoprotein in the blood-brain barrier of mice influences the brain penetration and pharmacological activity of many drugs. J Clin Invest 1996;97:2517-24.

32. Jetté L, Murphy GF, Leclerc JM, Beliveau R. Interaction of drugs with P-glycoprotein in brain capillaries. Biochem Pharmacol 1995;50:1701-9.

33. Goralski KB, Acott PD, Fraser AD, Worth D, Sinal CJ. Brain cyclosporin A levels are determined by ontogenic regulation of mdrla expression. Drug Metab Dispos 2006;34:288-95.

34. Dobbing J, Sands J. Comparative aspects of the brain growth spurt. Early Hum Dev 1979;3:79-83.

35. Lazarowski A, Caltana L, Merelli A, Rubio MD, Ramos AJ, Brusco A. Neuronal mdr-1 gene expression after experimental focal hypoxia: a new obstacle for neuroprotection? J Neurol Sci 2007;258:84-92.

36. Oh J, Kimchi-Sarfaty C, Kim I, Sherman A, Ambudkar S, Gottesman M. Functional characterization of SNP and haplotype of human MDR1 gene using a vaccinia virus expression system. Clin Pharmacol Ther 2005; 77:P24.

37. Nakamura T, Sakaeda T, Horinouchi M, et al. Effect of the mutation (C3435T) at exon 26 of the MDR1 gene on expression level of MDR1 messenger ribonucleic acid in duodenal enterocytes of healthy Japanese subjects. Clin Pharmacol Ther 2002;71:297-303. 